\title{
Aspectos epidemiológicos da tuberculose nas Regiões de Integração do estado do Pará, Brasil, no período entre 2005 e 2014
}

\section{Epidemiological aspects of tuberculosis in the Integration Regions of Pará State, Brazil, from 2005 to 2014}

Dilma Costa de Oliveira Neves', Luana de Oliveira Loureiro', Nathalya Pinheiro Paiva', Maria Deise de Oliveira Ohnishi', Cláudia Daniela Tourão Ribeiro'

' Centro Universitário do Estado do Pará, Belém, Pará, Brasil

\begin{abstract}
RESUMO
OBJETIVO: Descrever aspectos epidemiológicos da tuberculose (TB) em pacientes a partir de 20 anos de idade, residentes nas Regiões de Integração do estado do Pará, no período de 2005 a 2014. MATERIAIS E MÉTODOS: Estudo ecológico com base em dados do Sistema de Informação de Agravos de Notificação da Secretaria de Estado de Saúde Pública do Pará. As variáveis analisadas foram: sexo, raça/cor, idade, escolaridade, formas de TB, vulnerabilidade, uso de álcool, comorbidades, coinfecção pelo HIV e Região de Integração de residência. RESULTADOS: Foram estudados 35.926 casos na faixa etária a partir de 20 anos. A média de idade foi de $41,3( \pm 0,9)$ anos, com maior ocorrência em pessoas do sexo masculino $(63,1 \%)$ e cor parda $(71,5 \%)$. Houve predomínio do ensino fundamental, exceto na Região Metropolitana. Dentre as formas de TB, predominou a pulmonar (87,3\%). Entre os institucionalizados, a maior incidência foi na população carcerária $(47,4 \%)$. $\bigcirc$ uso de álcool $(38,6 \%)$ foi superior à presença de diabetes $(28,0 \%)$, coinfecção pelo HIV $(27,0 \%)$ e doença mental $(6,4 \%)$, com maior registro na Região Xingu $(79,9 \%)$. Observa-se uma convergência da incidência de TB e da proporção da coinfecção TB/HIV. CONCLUSÃO: Embora o Ministério da Saúde disponibilize esquemas terapêuticos eficazes para o tratamento da TB, os aspectos epidemiológicos analisados evidenciam que fatores, como os hábitos de vida, as comorbidades, a coinfecção pelo HIV, a baixa escolaridade e outros, contribuem para a manutenção da elevada incidência dessa doença no estado do Pará.
\end{abstract}

Palavras-chave: Tuberculose; Institucionalização; Coinfecção por HIV; Epidemiologia; Estudos Ecológicos.

\begin{abstract}
OBJECTIVE: To describe the epidemiological aspects of tuberculosis (TB) in patients $\geq 20$ years old living in the Integration Regions of Pará State, Brazil, from 2005 to 2014. MATERIALS AND METHODS: Ecological study based on data from the Information System on Diseases of Compulsory Declaration (Sinan) of the Secretariat of Public Health of Pará State (SESPA). The following variables were analyzed: gender, race/colour, age, schooling, forms of TB, vulnerability, alcohol consumption, comorbidities, HIV coinfection, and the Region of Integration of residence. RESULTS: A total of 35,926 cases were studied in the age group $\geq 20$ years old. The mean age was $41.3( \pm 0.9)$ years old, with the highest occurrence in males (63.1\%) and brown skin (71.5\%). There was a predominance of elementary education, except in the Metropolitan Region. Pulmonary form was the most predominant (87.3\%). Among institutionalized, the highest incidence was in the incarcerated population (47.4\%). Alcohol consumption (38.6\%) was more prevalent than diabetes (28.0\%), HIV coinfection (27.0\%), and mental illness (6.4\%), with highest occurrence in the Xingu Region (79.9\%). A convergence of TB incidence and the proportion of TB/HIV coinfection was observed. CONCLUSION: Although the Brazilian Ministry of Health provides effective therapeutic regimens for TB treatment, the epidemiological aspects analyzed showed that lifestyle, comorbidities, HIV coinfection, low schooling, and others factors contribute to the maintenance of high incidence of this disease in Pará State.
\end{abstract}

Keywords: Tuberculosis; Institutionalization; HIV Coinfection; Epidemiology; Ecological Studies.

\author{
Correspondência / Correspondence: \\ Dilma Costa de Oliveira Neves \\ Trav. Chaco, 2155. Bairro: Marco. CEP: 66093-572 - Belém, Pará, Brasil - Tel.: +55 (91) 99233-2525 \\ E-mail:dilmaconeves@gmail.com
}




\section{INTRODUÇÃO}

A tuberculose (TB) é uma doença antiga e sua existência é comprovada desde os tempos pré-históricos'. Dentre as formas clínicas, a pulmonar é considerada a mantenedora da elevada prevalência da doença na população. A forma primária pulmonar é mais comum nos casos diagnosticados em crianças. Embora a forma pós-primária seja mais comum em adultos jovens, também pode acometer qualquer faixa etária².

Por ser uma doença infecciosa de elevada magnitude, a TB tem sua importância mundial ${ }^{3}$, devido à elevada transmissibilidade do bacilo de Koch. A Organização Mundial da Saúde (OMS) estima que um terço da população mundial encontra-se infectada ${ }^{4}$.

Ainda segundo a OMS, o Brasil está na lista dos 20 países com elevada carga de $\mathrm{TB}^{5}$. No entanto, encontra-se também entre os países que estão avançando positivamente na redução da incidência de casos, juntamente com Indonésia, África do Sul, Tailândia e Vietnã ${ }^{6}$. No Brasil, no período de 2005 a 2014, foi diagnosticada uma média anual de 86.697 casos novos ${ }^{7}$ e 5.168 óbitos $^{8}$. Nesse período, houve uma redução da incidência, para todas as formas de TB, de 49,5/100.000 habitantes para 41,8/100.000 habitantes, com uma incidência média anual de 45,2/100.000 habitantes, o que corresponde a uma redução anual de $1,6 \%$. No estado do Pará, em igual período, foram registrados 40.990 casos novos de TB no Sistema de Informação de Agravos de Notificação (Sinan) e desses $87 \%$ foram notificados como forma pulmonar ${ }^{8}$.

Segundo o Programa Nacional de Controle da Tuberculose (PNCT), a Região Norte do Brasil possui 16 municípios considerados prioritários para a redução da incidência, e sete desses estão situados no estado do Pará9.

Alguns grupos populacionais, por apresentarem maior vulnerabilidade em adoecer, são considerados prioritários para o $\mathrm{PNCT}^{9}$. $\mathrm{O}$ risco de adoecimento por $T B$, nessas populações, é superior à média nacional para a população em geral. No grupo de pessoas privadas de liberdade, esse risco é considerado 25 vezes maior; entre os portadores de HIV, é de 30 vezes, e chega a 67 vezes maior na população em situação de rua ${ }^{10}$.

No Brasil, a condição que confere maior risco de morte a pessoas que vivem com HIV/aids é a TB ativa, e, na maioria das vezes, o diagnóstico da coinfecção pelo HIV ocorre por ocasião do diagnóstico ou do tratamento de $\mathrm{TB}^{11}$.

Embora tenha sido observada, no Brasil, uma redução do coeficiente de incidência de $T B$, foram diagnosticados 83.709 casos novos no ano de 20157, o que sugere a persistência dos desafios para a redução do número de casos da doença. Por isso, desde 2003, a TB encontra-se entre as doenças que se inserem nos critérios de doenças prioritárias pelo
Ministério da Saúde (MS). Por ser um dos cinco agravos de maior destaque no início deste século, a TB faz parte do Pacto pela Vida, do programa Mais Saúde, da Programação das Ações de Vigilância em Saúde, visando a operacionalização de ações que reduzam o seu impacto na morbimortalidade ${ }^{12}$.

Dessa forma, diante da problemática enfrentada atualmente pelo sistema de saúde pública no Pará, em decorrência de condições sociodemográficas favoráveis para a transmissão e desenvolvimento da TB, este estudo teve como objetivo descrever aspectos epidemiológicos dessa doença em pacientes a partir de 20 de idade, residentes nas Regiões de Integração do estado do Pará, no período de 2005 a 2014.

\section{MATERIAIS E MÉTODOS}

O estudo foi aprovado pelo Comitê de Ética em Pesquisa em Seres Humanos do Centro Universitário do Estado do Pará, sob o parecer de número 1.344.940, em $1^{\circ}$ de dezembro de 2015.

Trata-se de um estudo ecológico, com base em análise exploratória documental, utilizando dados de casos novos de TB em pessoas a partir de 20 anos de idade, notificados pelos serviços de saúde do estado do Pará, no período de 2005 a 2014, com registro no Sinan, sob gestão da Secretaria de Estado de Saúde Pública do Pará (SESPA)

Foram analisadas as seguintes variáveis existentes na ficha do Sinan: município de residência, sexo, raça/cor, idade, escolaridade, formas de TB, vulnerabilidade, uso de álcool, presença de diabetes, coinfecção pelo HIV e doença mental.

Foram excluídos os registros com idade e/ou município ignorados, assim como os registros com mais de $50 \%$ das variáveis eleitas para o estudo sem preenchimento.

O estudo foi realizado agrupando-se os 144 municípios constituintes do Estado nas 12 Regiões de Integração, a saber: Araguaia, Baixo Amazonas, Rio Caeté, Rio Capim, Carajás, Guamá, Lago de Tucuruí, Marajó, Metropolitana, Tapajós, Tocantins e Xingu ${ }^{13}$.

Os dados foram ajustados por idade, tanto entre as regionais como intrarregionalmente. Após o ajuste, os dados foram analisados pela estatística descritiva, com auxílio do programa BioEstat v5.4 ${ }^{14}$, utilizando-se o teste estatístico Qui-quadrado de aderência e aceito, como diferença significativa, o p-valor $<0,05$ para $\alpha<5 \%$.

\section{RESULTADOS}

Dentre os 40.990 casos de TB notificados no período do estudo, foram excluídos 613 (1,5\%) por não residirem no estado do Pará. Do total de 40.377 (98,5\%) casos notificados em residentes no Estado, 35.931 (89,0\%) encontravam-se na faixa de idade a partir de 20 anos, e desses foram estudados 35.926 casos com a confirmação de TB registrada 
nos arquivos do Sinan. Foram excluídos apenas cinco $(0,01 \%)$ casos que não continham o registro da confirmação de TB.

A raça/cor parda foi predominante $(71,5 \%)$ em todo - Estado, com maior proporção $(80,1 \%)$ observada na Região Baixo Amazonas. Houve predomínio do sexo masculino $(63,1 \%)$, com maior proporção na Região Xingu (68,8\%). A média de idade do Estado foi de $41,3( \pm 0,9)$ anos, não sendo observada diferença estatisticamente significativa entre as Regiões ( $p=0,9775)$. A média de idade, entre as Regiões, variou de $37,8( \pm 26,9)$ na Região Metropolitana a $44,3( \pm 16,4)$ anos na Região Araguaia. Houve predominância da faixa etária de 20 a 59 anos no Estado (84,9\%), com diferença estatística significativa $(p<0,0001)$ em relação à faixa etária a partir de 60 anos.

ensino fundamental predominou em mais de $50 \%$ dos casos, com exceção da proporção de 47,4\% observada na Região Metropolitana (Tabela 1).

A forma pulmonar predominou ( $p<0,0001)$ em relação as outras formas de TB (87,3\%), tanto no Estado como em suas Regiões. A segunda maior proporção observada foi a forma extrapulmonar, sendo que a Região Metropolitana apresentou um valor proporcional $(11,9 \%)$ superior ao do Estado (9,9\%) (Tabela 2).

$\bigcirc$ uso de álcool foi mais prevalente que diabetes, doença mental e coinfecção pelo HIV, tanto nas Regiões como no Estado. A diabetes foi mais prevalente no Estado e nas Regiões Baixo Amazonas, Guamá, Marajó, Tocantins, Lago de Tucuruí, Carajás e Xingu, enquanto, nas demais Regiões, houve maior quantidade de registros para a coinfecção pelo HIV (Tabela 3).

$\mathrm{Na}$ tabela 4, observa-se que 6,0\% (2.164/35.926) dos casos notificados se encontravam institucionalizados; dentre esses, predominou os que estavam em presídios $(47,4 \%)$.

Observou-se, no período estudado, um incremento de $171,7 \%(14,4-5,3 / 5,3)$ na proporção de casos de coinfecção TB/HIV em relação ao total de casos de TB por todas as formas, enquanto que, para a incidência de TB, houve um declínio de 13,5\% $(90,2-78,0 / 90,2)$ (Gráfico 1).

Tabela 1 - Distribuição dos casos de TB em pacientes a partir de 20 anos de idade, segundo a escolaridade, notificados nas Regiões de Integração do estado do Pará, Brasil, de 2005 a 2014

\begin{tabular}{|c|c|c|c|c|c|c|c|c|c|c|c|}
\hline \multirow{2}{*}{$\begin{array}{l}\text { Região de } \\
\text { Integração }\end{array}$} & \multicolumn{2}{|c|}{ Analfabeto } & \multicolumn{2}{|c|}{ Ensino fundamental } & \multicolumn{2}{|c|}{ Ensino médio } & \multicolumn{2}{|c|}{ Ensino superior } & \multicolumn{2}{|c|}{ Ignorado } & \multirow{2}{*}{ Total } \\
\hline & $\mathrm{N}$ & $\%$ & $\mathrm{~N}$ & $\%$ & $\mathrm{~N}$ & $\%$ & $\mathrm{~N}$ & $\%$ & N & $\%$ & \\
\hline Araguaia* & 150 & 14,1 & 582 & 54,8 & 94 & 8,8 & 18 & 1,7 & 219 & 20,6 & 1.063 \\
\hline Baixo Amazonas* & 169 & 8,1 & 1.157 & 55,6 & 452 & 21,7 & 90 & 4,3 & 215 & 10,3 & 2.083 \\
\hline Carajás* & 241 & 13,5 & 1.043 & 58,5 & 316 & 17,7 & 74 & 4,2 & 109 & 6,1 & 1.783 \\
\hline Guamá* & 170 & 5,6 & 1.805 & 59,7 & 416 & 13,7 & 52 & 1,7 & 584 & 19,3 & 3.027 \\
\hline Lago de Tucuruí & 169 & 16,8 & 592 & 58,8 & 105 & 10,4 & 26 & 2,6 & 115 & 11,4 & 1.007 \\
\hline Marajó* & 191 & 17,2 & 614 & 55,2 & 105 & 9,4 & 29 & 2,6 & 174 & 15,6 & 1.113 \\
\hline Metropolitana* & 493 & 2,7 & 8.720 & 47,4 & 4.347 & 23,7 & 1.040 & 5,7 & 3.775 & 20,5 & 18.375 \\
\hline Rio Caeté ${ }^{\dagger}$ & 165 & 9,7 & 927 & 54,4 & 231 & 13,5 & 37 & 2,2 & 345 & 20,2 & 1.705 \\
\hline Rio Capim* & 211 & 13,5 & 866 & 55,6 & 177 & 11,3 & 17 & 1,1 & 288 & 18,5 & 1.559 \\
\hline Tapajós* & 180 & 19,6 & 535 & 58,3 & 96 & 10,5 & 12 & 1,3 & 94 & 10,3 & 917 \\
\hline Tocantins* & 217 & 9,3 & 1.303 & 55,6 & 302 & 12,9 & 60 & 2,5 & 462 & 19,7 & 2.344 \\
\hline Xingu $^{\dagger}$ & 116 & 12,2 & 484 & 51,0 & 100 & 10,5 & 24 & 2,5 & 226 & 23,8 & 950 \\
\hline Estado $\ddagger$ & 2.472 & 6,9 & 18.628 & 51,8 & 6.741 & 18,8 & 1.479 & 4,1 & 6.606 & 18,4 & 35.926 \\
\hline
\end{tabular}


Tabela 2 - Distribuição dos casos de TB em pacientes a partir de 20 anos de idade, segundo a forma, notificados nas Regiões de Integração do estado do Pará, Brasil, de 2005 a 2014

\begin{tabular}{|c|c|c|c|c|c|c|c|c|c|}
\hline \multirow{2}{*}{ Região de Integração } & \multicolumn{2}{|c|}{ Pulmonar } & \multicolumn{2}{|c|}{ Extrapulmonar } & \multicolumn{2}{|c|}{$\begin{array}{l}\text { Pulmonar + } \\
\text { extrapulmonar }\end{array}$} & \multicolumn{2}{|c|}{ Não informado } & \multirow{2}{*}{ Total } \\
\hline & $\mathrm{N}$ & $\%$ & $\mathrm{~N}$ & $\%$ & $N$ & $\%$ & $\mathrm{~N}$ & $\%$ & \\
\hline Araguaia* & 957 & 90,0 & 84 & 7,9 & 21 & 2,0 & 1 & 0,1 & 1.063 \\
\hline Baixo Amazonas* & 1.799 & 86,4 & 201 & 9,6 & 83 & 4,0 & - & - & 2.083 \\
\hline Caraiás* & 1.595 & 89,4 & 144 & 8,1 & 44 & 2,5 & - & - & 1.783 \\
\hline Guamá* & 2.770 & 91,5 & 204 & 6,7 & 50 & 1,7 & 3 & 0,1 & 3.027 \\
\hline Lago de Tucuruí & 894 & 88,8 & 95 & 9,4 & 17 & 1,7 & 1 & 0,1 & 1.007 \\
\hline Marajó* & 1.024 & 92,0 & 66 & 5,9 & 23 & 2,1 & - & - & 1.113 \\
\hline Metropolitana* & 15.572 & 84,7 & 2.182 & 11,9 & 620 & 3,4 & 1 & - & 18.375 \\
\hline Rio Caeté* & 1.545 & 90,6 & 127 & 7,4 & 30 & 1,8 & 3 & 0,2 & 1.705 \\
\hline Rio Capim* & 1.423 & 91,3 & 103 & 6,6 & 33 & 2,1 & - & - & 1.559 \\
\hline Tapajós* & 835 & 91,0 & 62 & 6,8 & 20 & 2,2 & - & - & 917 \\
\hline Tocantins* & 2.085 & 89,0 & 210 & 8,9 & 38 & 1,6 & 11 & 0,5 & 2.344 \\
\hline Xingu* & 873 & 91,9 & 72 & 7,6 & 5 & 0,5 & - & - & 950 \\
\hline Estado* & 31.372 & 87,3 & 3.550 & 9,9 & 984 & 2,7 & 20 & 0,1 & 35.926 \\
\hline
\end{tabular}

Qui-quadrado de aderência para proporções esperadas iguais; ${ }^{*} p<0,0001$; Sinal convencional utilizado: - Dado numérico igual a zero, não resultante de arredondamento.

Tabela 3 - Distribuição, segundo a presença de diabetes, coinfecção pelo HIV, doença mental e uso de álcool, dos casos de TB em pessoas a partir de 20 anos de idade, notificados nas Regiões de Integração do estado do Pará, Brasil, de 2005 a 2014

\begin{tabular}{|c|c|c|c|c|c|c|c|c|c|}
\hline \multirow{2}{*}{ Região de Integração } & \multicolumn{2}{|c|}{ Uso de álcool } & \multicolumn{2}{|c|}{ Diabetes } & \multicolumn{2}{|c|}{ Coinfecção pelo HIV } & \multicolumn{2}{|c|}{ Doença mental } & \multirow{2}{*}{ Total } \\
\hline & $\mathrm{N}$ & $\%$ & $\mathrm{~N}$ & $\%$ & $\mathrm{~N}$ & $\%$ & $\mathrm{~N}$ & $\%$ & \\
\hline Araguaia & 65 & 42,0 & 36 & 23,2 & 40 & 25,8 & 14 & 9,0 & 155 \\
\hline Baixo Amazonas & 205 & 39,2 & 170 & 32,5 & 113 & 21,6 & 35 & 6,7 & 523 \\
\hline Carajás & 216 & 47,0 & 110 & 23,9 & 103 & 22,4 & 31 & 6,7 & 460 \\
\hline Guamá & 252 & 38,0 & 212 & 31,9 & 148 & 22,3 & 52 & 7,8 & 664 \\
\hline Lago de Tucuruí & 101 & 39,2 & 64 & 24,8 & 61 & 23,6 & 32 & 12,4 & 258 \\
\hline Marajó & 88 & 42,3 & 63 & 30,3 & 35 & 16,8 & 22 & 10,6 & 208 \\
\hline Metropolitana & 1.913 & 33,5 & 1.710 & 30,0 & 1.760 & 30,9 & 319 & 5,6 & 5.702 \\
\hline Rio Caeté & 124 & 46,8 & 34 & 12,8 & 82 & 31,0 & 25 & 9,4 & 265 \\
\hline Rio Capim & 188 & 47,6 & 84 & 21,3 & 96 & 24,3 & 27 & 6,8 & 395 \\
\hline Tapajós & 98 & 47,3 & 44 & 21,3 & 50 & 24,2 & 15 & 7,2 & 207 \\
\hline Tocantins & 179 & 44,1 & 112 & 27,6 & 88 & 21,7 & 27 & 6,6 & 406 \\
\hline Xingu & 271 & 79,9 & 45 & 13,3 & 15 & 4,4 & 8 & 2,4 & 339 \\
\hline Estado & 3.700 & 38,6 & 2.684 & 28,0 & 2.591 & 27,0 & 607 & 6,4 & 9.582 \\
\hline
\end{tabular}


Tabela 4 - Distribuição, segundo a condição de institucionalizado, dos casos de TB em pacientes a partir de 20 anos de idade, notificados nas Regiões de Integração do estado do Pará, Brasil, de 2005 a 2014

\begin{tabular}{|c|c|c|c|c|c|c|c|c|c|c|}
\hline \multirow{2}{*}{ Região de Integração } & \multicolumn{2}{|c|}{ Presídio } & \multicolumn{2}{|c|}{ Asilo } & \multicolumn{2}{|c|}{ Hospital psiquiátrico } & \multicolumn{2}{|c|}{ Outro* } & \multicolumn{2}{|c|}{ Total } \\
\hline & $\mathrm{N}$ & $\%$ & $N$ & $\%$ & $\mathrm{~N}$ & $\%$ & $\mathrm{~N}$ & $\%$ & $\mathrm{~N}$ & $\%$ \\
\hline Araguaia & 23 & 46,0 & - & - & 1 & 2,0 & 26 & 52,0 & 50 & 2,3 \\
\hline Baixo Amazonas & 63 & 53,0 & - & - & 1 & 0,8 & 55 & 46,2 & 119 & 5,5 \\
\hline Carajás & 43 & 39,1 & - & - & 1 & 0,9 & 66 & 60,0 & 110 & 5,1 \\
\hline Guamá & 111 & 52,6 & 2 & 1,0 & - & - & 98 & 46,4 & 211 & 9,7 \\
\hline Lago de Tucuruí & 22 & 36,7 & 2 & 3,3 & 1 & 1,7 & 35 & 58,3 & 60 & 2,8 \\
\hline Marajó & 38 & 50,0 & 3 & 4,0 & 1 & 1,3 & 34 & 44,7 & 76 & 3,5 \\
\hline Metropolitana & 507 & 48,7 & 16 & 1,5 & 6 & 0,6 & 512 & 49,2 & 1.041 & 48,1 \\
\hline Rio Caeté & 58 & 48,4 & 1 & 0,8 & 1 & 0,8 & 60 & 50,0 & 120 & 5,5 \\
\hline Rio Capim & 39 & 42,4 & 4 & 4,3 & - & - & 49 & 53,3 & 92 & 4,3 \\
\hline Tapajós & 27 & 35,1 & 1 & 1,3 & - & - & 49 & 63,6 & 77 & 3,6 \\
\hline Tocantins & 61 & 50,0 & 2 & 1,6 & - & - & 59 & 48,4 & 122 & 5,6 \\
\hline Xingu & 33 & 38,4 & 1 & 1,2 & - & - & 52 & 60,4 & 86 & 4,0 \\
\hline Estado & 1.025 & 47,4 & 32 & 1,5 & 12 & 0,5 & 1.095 & 50,6 & 2.164 & 100,0 \\
\hline
\end{tabular}

Sinal convencional utilizado: - Dado numérico igual a zero, não resultante de arredondamento; ${ }^{*}$ Institucionalização não especificada na ficha do Sinan.

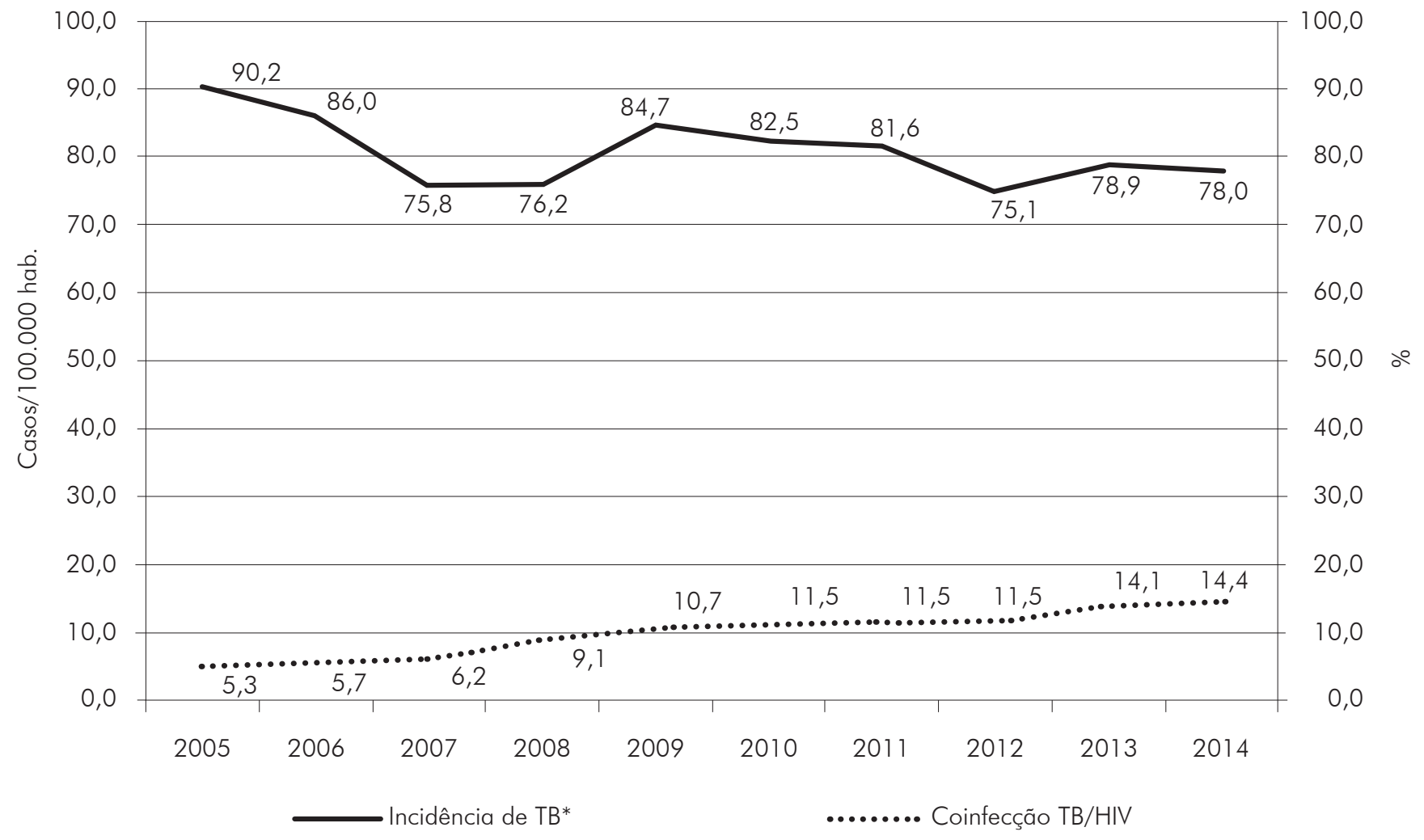

Fonte: Sinan, SESPA

* Coeficiente por 100.000 pessoas a partir de 20 anos de idade.

Gráfico 1 - Tendências de incidência de TB e proporção da coinfecção TB/HIV em pacientes a partir de 20 anos de idade no estado do Pará, Brasil, de 2005 a 2014 


\section{DISCUSSÃO}

A meta prevista pela OMS é a eliminação da TB até o ano de 2050, ou seja, que a taxa de incidência seja menor que um caso a cada 1 milhão de habitantes. Essa proposta tem como enfrentamento a elevada magnitude da TB em regiões e em países em desenvolvimento, como o Brasil, apesar do avanço da medicina e do desenvolvimento de novas tecnologias ${ }^{11}$.

Os aspectos epidemiológicos estudados permitiram identificar, no Pará, um predomínio do adoecimento em homens $(63,1 \%)$, com maior concentração na faixa etária de 20 a 59 anos de idade $(84,9 \%)$ e na raça/cor parda $(71,5 \%)$. Segundo dados do $M S^{9}$, no Brasil observa-se uma maior proporção de homens com TB, assim como foi constatado em outros dois estudos, um realizado no estado do Mato Grosso do Sul (66,3\%) no período de 2001 a $2009{ }^{15}$ e outro, no município de Patos (66,9\%), estado da Paraíba, no período de 2001 a $2010^{16}$. No país, em 2016, o risco de adoecimento por TB na população masculina, a partir 20 anos de idade $(80,1 / 100.000$ habitantes), foi 2,5 vezes maior que em mulheres $(31,9 / 100.000 \text { habitantes) })^{7}$.

Esses achados podem ser em decorrência da posição assumida pelo homem na sociedade, como o de ser o provedor de seu lar; além das barreiras institucionais que restringem os horários de funcionamento dos serviços de saúde aos de trabalho e nem sempre as demandas são resolvidas em uma única consulta ${ }^{17}$. Além disso, a maior incidência em homens, como portadores majoritários da $\mathrm{TB}$ em relação às mulheres, pode ser justificada também devido à maior prevalência de infecção pelo HIV, etilismo e uso abusivo de drogas, condições que os tornam mais vulneráveis à infecção e ao adoecimento pela doença ${ }^{18}$.

Sabe-se que a construção da identidade masculina possui uma subjetividade própria do gênero, o que possibilita aos homens considerar a doença como um sinal de fragilidade, pois se julgam invulneráveis a esses agravos, contribuindo para um menor autocuidado e uma maior exposição a situações de risco. Sendo assim, o homem protela a procura de atendimento, permitindo o agravamento dos casos, o sofrimento físico e emocional de si e de seus familiares, além de maiores despesas próprias e para o Sistema Único de Saúde, intervindo nas fases mais avançadas da doença ${ }^{19}$.

maior número de casos de TB registrados em homens na Região do Xingu pode estar relacionado com a instalação de empresas, como a Usina Hidrelétrica de Belo Monte no Rio Xingu, demandando, provavelmente, movimentos migratórios em busca de trabalho sob condições adversas nos principais municípios dessa Região; além disso, a maioria desses municípios possui baixo Índice de Desenvolvimento Humano $(\text { IDH })^{20}$.

A ocorrência de casos na faixa de idade entre 20 e 59 anos $(84,9 \%)$, constatada por este estudo, foi superior ao observado no estado de São Paulo, onde quase $70,0 \%$ dos casos novos ocorreram nessa faixa etária $^{21}$, assim como em outros estudos, como os realizados em Recife $(78,6 \%)$, estado de Pernambuco ${ }^{22}$, e em Belo Horizonte $(78,1 \%)$, estado de Minas Gerais ${ }^{23}$. Essas proporções mostram que muitos pacientes podem enfrentar sérios problemas no sustento de suas famílias $^{21}$.

No Brasil, a TB atinge predominantemente pessoas de 20 a 49 anos de idade; cerca de 63\% dos casos novos da doença, em 2009, encontrava-se nessa faixa etária ${ }^{10}$. A variabilidade da média de idade pode denotar a presença de desigualdades sociais entre as Regiões de Integração do Pará, acometendo populações mais jovens, como observado na Região Metropolitana com 37,8 $( \pm 26,9)$ anos. Um estudo realizado em Ribeirão Preto, estado de São Paulo, no período de 2006 a 2009, demonstrou que a TB continua a ser uma doença fortemente relacionada com as características sociais de uma população ${ }^{24}$.

Ressalta-se que os resultados obtidos na análise de um indicador indireto das condições sociais, como - baixo nível de escolaridade, refletem a relevância do contexto socioeconômico para o controle da TB, influenciando na maneira como o indivíduo afetado terá o desenvolvimento de seu autocuidado frente às orientações para o tratamento e a prevenção da doença ${ }^{25}$. A baixa escolaridade pode um fator relevante, pois a mesma comprometeria a compreensão da doença e de seu tratamento por parte do doente e da população; por isso, a implementação do tratamento diretamente observado pode ser decisivo para o alcance de bons resultados ${ }^{26}$.

A busca ativa de sintomáticos respiratórios consiste na principal estratégia que deve ser priorizada pelos serviços de saúde para a identificação de casos de TB em sua forma pulmonar; isso porque $90 \%$ dos casos da doença são da forma pulmonar e desses 60\% são bacilíferas $^{12}$. Esse predomínio da forma pulmonar, no Pará e em suas Regiões, também foi observado em estudos semelhantes; um deles foi realizado no estado de Alagoas, detectando $86,8 \%{ }^{27}$, e outro, em Gurupi, no estado de Tocantins, entre 2005 e 2010, com $88,7 \%$ dos casos na forma pulmonar ${ }^{28}$. Tais dados reforçam a importância da adequada organização do trabalho nas unidades básicas de saúde, a qual deve possibilitar não apenas a suspeição e o diagnóstico da doença, mas também assegurar um fluxo de atendimento resolutivo para os casos suspeitos e confirmados, além da busca ativa de sintomáticos respiratórios na comunidade e entre os contatos dos casos índice ${ }^{29}$.

$\bigcirc$ uso de álcool foi condição presente e superior à coinfecção pelo HIV em todas as Regiões estudadas e em maior proporção na Região Xingu. A diabetes e a doença mental também foram comorbidades presentes neste estudo. Piva et al. ${ }^{30}$ observaram que $55,2 \%$ dos pacientes portadores de TB pulmonar faziam uso de álcool e 24,1\% apresentavam diabetes como a principal comorbidade. Outra pesquisa, realizada em Minas Gerais, encontrou o uso de álcool em $17,2 \%$ dos casos e diabetes em 3,8\%31. $\bigcirc$ achado 
de elevadas proporções de pacientes que fazem uso de álcool, nas diferentes Regiões do Pará, pode ser devido à presença de fatores de risco relacionados ao meio em que vivem. Esses fatores relacionam-se tanto ao meio familiar desestruturado como à própria ausência da família, assim como a baixa perspectiva de oferta de emprego para uma mão de obra com baixa escolaridade, condições essas que fazem com que ocorra a busca pela bebida como meio de fuga para seus problemas ${ }^{15,32}$

A presença de doenças ou de situações que propiciem o declínio do estado de defesa do organismo, tais como diabetes, neoplasias, desnutrição, HIV, uso de corticoides, uso de álcool, entre outros, compromete o organismo do paciente oferecendo condições favoráveis à proliferação do bacilo ${ }^{12,33}$. $\bigcirc$ reconhecimento desses fatores de risco, no primeiro contato com o paciente, possibilita a identificação de medidas que intensifiquem o acompanhamento e a instituição do tratamento diretamente observado, a fim de minimizar, ou mesmo evitar, o abandono do esquema, o uso indevido das medicações e a reativação da doença ${ }^{33}$.

A população em situação de rua tem sua vulnerabilidade, por apresentar, em sua maioria, comorbidades como desnutrição, doenças cardiovasculares, transtornos mentais, dependência química e infecções sexualmente transmissíveis ${ }^{12}$.

$\bigcirc$ achado de $47,4 \%$ de casos de TB em pessoas privadas de liberdade foi superior ao observado em dois estudos realizados no estado do Rio Grande do Norte, um em Parnamirim $(3,3 \%)^{34}$ e outro em São Gonçalo do Amarante $(2,0 \%)^{35}$. Sabe-se que nessa população é maior o risco de exposição ao bacilo de Koch, de infecção e de desenvolvimento da doença, devido às condições das celas mal ventiladas e à superlotação dos presídios brasileiros ${ }^{35}$.

A institucionalização de pessoas em condições desfavoráveis à manutenção da saúde, como nos presídios, asilos e outros, exige que os serviços de saúde existentes nesses locais sejam adequados, visando reduzir a transmissão do bacilo da TB com a identificação precoce de casos suspeitos, além de assegurar os cuidados voltados à saúde ocupacional dos trabalhadores desses locais ${ }^{12}$.

A população carcerária, assim como as pessoas em situação de rua, os pacientes com TB coinfectados pelo HIV, os indígenas e os profissionais de saúde constituem alguns dos grupos populacionais com maior vulnerabilidade de adoecimento pelo bacilo de Koch e, por isso, são considerados como população prioritária pelo $\mathrm{PNCT}^{9}$.

A convergência das epidemias de TB e HIV é um dos maiores desafios para a saúde pública no mundo; e, em decorrência das implicações da coinfecção $\mathrm{TB} / \mathrm{HIV}$, é recomendado que o teste anti-HIV (rápido) seja oferecido precocemente aos indivíduos com diagnóstico estabelecido de TB, independente da confirmação bacteriológica e preferencialmente pela rede da atenção básica ${ }^{12}$.

$\mathrm{Na}$ contramão do declínio da incidência de casos de TB, no período estudado, está o crescimento linear da coinfecção TB/HIV, o que pode ainda coexistir com a subnotificação dos casos, uma vez que as pessoas com HIV/aids estão mais suscetíveis à infecção pelo bacilo de Koch. Esse achado reforça a necessidade de maiores e melhores investimentos na atenção primária à saúde, principalmente no diagnóstico precoce de HIV em pacientes com TB e no diagnóstico de TB em pacientes com HIV 36 .

No Pará, além do baixo IDH na maioria de seus municípios $^{20}$, a cobertura da Estratégia Saúde da Família varia de 15,1\% na Região Xingu a 48,4\% na Região Carajás, o que representa baixa cobertura populacional, resultando na pouca detecção de casos e a reduzida assistência necessária à população pela Atenção Primária em Saúde ${ }^{37}$.

Algumas limitações decorridas neste estudo estão relacionadas com o preenchimento da ficha de notificação. A versão da ficha que serviu como instrumento de coleta de dados ainda não possui variáveis específicas para algumas das populações listadas pelo MS, como a de vulnerabilidade à TB. Apesar da existência de tais limitações, as contribuições apresentadas podem aperfeiçoar as ações do Programa de Controle da Tuberculose nas Regiões de Integração do Pará, a fim de dar relevância a alguns estados de vulnerabilidade existentes na população, assim como apontar as Regiões que necessitam de reorganização da Atenção Primária em Saúde e a magnitude da TB como problema de saúde pública no Pará.

\section{CONCLUSÃO}

Os aspectos epidemiológicos analisados permitem evidenciar que, embora o MS disponibilize esquemas terapêuticos comprovadamente eficazes para o tratamento da TB, a existência de fatores, como os hábitos de vida, as comorbidades, a coinfecção pelo HIV, a baixa escolaridade e outros, contribuem para a manutenção da elevada incidência dessa doença no estado do Pará.

\section{CONFLITOS DE INTERESSE}

Os autores declaram não ter havido conflitos de interesse por parte dos mesmos e da SESPA.

\section{CONTRIBUIÇÃO DOS AUTORES}

Todos os autores contribuíram com a idealização do estudo, a análise e a interpretação dos dados e com a redação do manuscrito, aprovando a versão final publicada. Declaram-se responsáveis pelo conteúdo integral do artigo, garantindo sua precisão e integridade. 


\section{REFERÊNCIAS}

1 Gonçalves BD. Perfil epidemiológico da exposição à tuberculose em um hospital universitário: uma proposta de monitoramento da doença [dissertação]. Rio de Janeiro: Fiocruz; 2009.

2 Bertolozzi MR, Takahashi RF, Hino P, Litvoc M, França FOS. $\bigcirc$ controle da tuberculose: um desafio para a saúde pública. Rev Med (São Paulo). $2014 ; 93(2): 83-9$.

3 Ministério da Saúde (BR). Secretaria de Vigilância em Saúde. Detectar, tratar e curar: desafios e estratégias brasileiras frente à tuberculose. Bol Epidemiol. 2015;46(9): 1 - 19.

4 World Health Organization. Global tuberculosis report: 2014 [Internet]. Geneva: WHO; 2014 [cited 2015 Feb 20]. Available from: http://apps.who.int/ medicinedocs/en/d/Js21634en/.

5 World Health Organization. Global tuberculosis report: 2016 [Internet]. Geneva: WHO; 2016 [cited 2017 Jan 11]. Available from: http://apps.who.int/ medicinedocs/en/d/Js23098en/.

6 World Health Organization. Global tuberculosis report: 2017 [Internet]. Geneva: WHO; 2017 [cited 2017 Sep 18]. Available from: http://www.who.int/ tb/publications/global_report/en/.

7 Ministério da Saúde (BR). Departamento de Informática do Sistema Único de Saúde. TabNet Win32 3.0: Tuberculose - Casos confirmados notificados no Sistema de Informação de Agravos de Notificação - Brasil [Internet]. Brasília: Datasus; 2017 [citado 2017 set 18]. Disponível em: http:// tabnet.datasus.gov.br/cgi/tabcgi.exe? sinannet/cnv/ tubercbr.def.

8 Ministério da Saúde (BR). Departamento de Informática do Sistema Único de Saúde. TabNet Win32 3.0: Mortalidade - Brasil [Internet]. Brasília: Datasus; 2017 [citado 2017 set 18]. Disponível em: http://tabnet. datasus.gov.br/cgi/tabcgi.exe? sim/cnv/obtl 0 uf.def.

9 Ministério da Saúde (BR). Secretaria de Vigilância em Saúde. Departamento de Vigilância das Doenças Transmissíveis. Panorama da tuberculose no Brasil: indicadores epidemiológicos e operacionais. Brasília: Ministério da Saúde; 2014. 92 p.

10 Piller RVB. Epidemiologia da tuberculose. Pulmao RJ. 2012;21(1):4-9.

11 World Health Organization. Global tuberculosis report. 20th ed. Geneva: WHO; 2015. 192 p.

12 Ministério da Saúde (BR). Secretaria de Vigilância em Saúde. Departamento de Vigilância Epidemiológica. Manual de recomendações para o controle da tuberculose no Brasil. Brasília: Ministério da Saúde; 2011. 284 p. (Série A. Normas e manuais técnicos).
13 Brasil. Ministério Público Federal. Procuradoria da República no Pará. Decreto Estadual n. 1.066, de 19 de junho de 2008. Dispõe sobre a regionalização do Estado do Pará e dá outras providências [Internet]. 2008 [citado 2015 abr 22]. Disponível em: http://www.prpa.mpf.gov.br/ setorial/biblioteca/legislacao/decreto-estadual-n-1 066-de-19-de-junho-de-2008.

14 Ayres M, Ayres Jr MA, Ayres DL, Santos AS. BioEstat 5.4: aplicações estatísticas nas áreas das ciências biológicas e médicas. Belém: UFPA; 2014. (Software).

15 Basta PC, Marques M, Oliveira RL, Cunha EAT, Resendes APC, Souza-Santos R. Desigualdades sociais e tuberculose: análise segundo raça/cor, Mato Grosso do Sul. Rev Saude Publica. 2013 out;47(5):854-64.

16 Araujo KMFA, Figueiredo TMRM, Gomes LCF, Pinto ML, Silva TC, Bertolozzi MR. Evolução da distribuição espacial dos casos novos de tuberculose no município de Patos (PB), 2001-2010. Cad Saude Colet. 2013 jul-set;21 (3):296-302.

17 Beraldo AA, Arakawa T, Pinto ESG, Andrade RLP, Wysocki AD, Silva Sobrinho RA, et al. Atraso na busca por serviço de saúde para o diagnóstico da tuberculose em Ribeirão Preto (SP). Cienc Saude Coletiva. 2012 nov; 17(11):3079-86.

18 Oliveira Junior HS, Mendes DHC, Almeida RB. Prevalência de casos de tuberculose durante os anos de 2002 a 2012, no município de Palmas-Paraná, Brasil. Rev Saude Publica St. Catarina. 2015 jan-abr;8(1):43-57.

19 Braz N. A construção da subjetividade masculina e seu impacto sobre a saúde do homem: reflexão bioética sobre justiça distributiva. Cienc Saude Coletiva. 2005 jan-mar; 10(1):97-104.

20 Programa das Nações Unidas para o Desenvolvimento. Instituto de Pesquisa Econômica Aplicada. Fundação João Pinheiro. Atlas de desenvolvimento humano no Brasil: índice de desenvolvimento humano municipal brasileiro [Internet]. 2010 [citado 2017 abr 22]. Disponível em: http://atlasbrasil.org.br/2013/pt/ranking\&gt.

21 Secretaria de Estado da Saúde de São Paulo (São Paulo). Centro de Vigilância Epidemiológica. Coordenadoria de Controle de Doenças. Alguns aspectos epidemiológicos do controle da tuberculose no estado de São Paulo. BE CVE. 2012 jun;2(1 1):177-99.

22 Silva CCAV, Andrade MS, Cardoso MD. Fatores associados ao abandono do tratamento de tuberculose em indivíduos acompanhados em unidades de saúde de referência na cidade do Recife, Estado de Pernambuco, Brasil, entre 2005 e 2010. Epidemiol Serv Saude. 2013 mar;22(1):77-85. 
23 Reis DC, Almeida TAC, Quites HFO, Sampaio MM. Epidemiological profile of tuberculosis in the city of Belo Horizonte (MG), from 2002 to 2008. Rev Bras Epidemiol. 2013 sep;16(3):592-602.

24 Roza DL, Caccia-Bava MCGG, Martinez EZ. Spatio-temporal patterns of tuberculosis incidence in Ribeirão Preto, State of São Paulo, southeast Brazil, and their relationship with social vulnerability: a Bayesian analysis. Rev Soc Bras Med Trop. 2012 Sep-Oct:45(5):607-15.

25 Monteiro NLS, Luna Neto RT, Tavares NBF, Campos $\mathrm{RI}$, Alencar AFO, Lima MAS, et al. Abandono do tratamento da tuberculose: uma análise epidemiológica dos seus fatores de risco. Cad Cult Cienc. 2015 mar;13(2).

26 Figueiredo TMRM, Silva TC, Pinto ML, Silva EL, Araújo KMFA. Avaliação dos serviços de atenção secundária e primária à saúde no controle da tuberculose, município de Campina Grande-PB-Brasil. In: Anais do $2^{\circ}$ Congresso Brasileiro de Política, Planejamento e Gestão em Saúde [Internet]; 2013 out 1-3; Belo Horizonte, MG. Rio de Janeiro: Abrasco; 2013 [citado 2015 abr 25]. Disponível em: http://www. politicaemsaude.com.br/download/download?ID_ DOWNLOAD $=261$.

27 Silva EG, Vieira JDS, Cavalcante AL, Santos LGML, Rodrigues APRA, Cavalcante TCS. Perfil epidemiológico da tuberculose no estado de Alagoas de 2007 a 2012. Cad Grad Cienc Biol Saude. 2015 nov;3(1):31-45.

28 Kamimura QP, Gonçalves KAM, Silva JLG. Caracterização do perfil epidemiológico e sócio-demográfico de cidadãos portadores de tuberculose. Ens Cienc Cienc Biol Agrar Saude. 2012;16(6):119-28.

29 Villa TCS, Ponce MAZ, Wysocki AD, Andrade RLP, Arakawa T, Scatolin BE, et al. Diagnóstico oportuno da tuberculose nos serviços de saúde de diversas regiões do Brasil. Rev Latino-Am Enfermagem. 2013 jan-fev;21 (esp): 1 -8.
30 Piva SGN, Costa MCN, Barreto FR, Pereira SM. Prevalência de deficiência nutricional em pacientes com tuberculose pulmonar. J Bras Pneumol. 2013 jul-ago;39(4):476-83.

31 Augusto CJ, Carvalho WS, Gonçalves AD, Ceccato MGB, Miranda SS. Características da tuberculose no estado de Minas Gerais entre 2002 e 2009. J Bras Pneumol. 2013 mai-jun;39(3):357-64.

32 Marques ACPR, Cruz MS. O adolescente e o uso de drogas. Rev Bras Psiquiatr. 2000 dez;22 Supl 2:32-6.

33 Oliveira HB, Moreira Filho DC. Recidivas em tuberculose e seus fatores de risco. Rev Panam Salud Publica. 2000;7(4):232-41.

34 Barbosa IR, Henrique GL. Caracterização dos casos de tuberculose em um município prioritário no estado do Rio Grande do Norte. Rev APS. 2014 jan-mar; 17(1):24-31.

35 Barbosa IR, Silva JL. Dados epidemiológicos da tuberculose em São Gonçalo do Amarante - RN. Rev Bras Cienc Saude. 2013 abr-jun; 11 (36):29-37.

36 Ministério da Saúde (BR). Secretaria de Vigilância em Saúde. O controle da tuberculose no Brasil: avanços, inovações e desafios. Bol Epidemiol. $2014 ; 45(2): 1-13$.

37 Ministério da Saúde (BR). Departamento de Informática do Sistema Único de Saúde. TabNet Win32 3.0: Projeção da população das unidades da federação por sexo e grupos de idade: 2000-2030 [Internet]. Brasília: Datasus; 2017 [citado 2017 set 18]. Disponível em: http:// tabnet.datasus.gov.br/cgi/tabcgi.exe?ibge/cnv/ projpopuf.def. 